\title{
Cognitive Retraining in Traumatic Brain Injury: Experience from Tertiary Care Center in Southern India
}

\author{
Mohammed Afsar ${ }^{1}$ Dhaval Shukla ${ }^{2}$ Binukumar Bhaskarapillai ${ }^{3} \quad$ Jamuna Rajeswaran ${ }^{1}$ \\ ${ }^{1}$ Department of Clinical Psychology, National Institute of Mental \\ Health and Neuro Sciences, Bengaluru, Karnataka, India \\ 2Department of Neurosurgery, National Institute of Mental Health \\ and Neuro Sciences, Bengaluru, Karnataka, India \\ ${ }^{3}$ Department of Biostatistics, National Institute of Mental Health \\ Address for correspondence Jamuna Rajeswaran, MPhil, \\ PhD, Department of Clinical Psychology, M.V. Govindaswamy \\ Centre, National Institute of Mental Health and Neuro Sciences \\ (NIMHANS), 3rd Floor, Bengaluru 560029, Karnataka, India \\ (e-mail: drjamunarajan@gmail.com).
} and Neuro Sciences, Bengaluru, Karnataka, India

J Neurosci Rural Pract 2021;12:295-301.

\begin{abstract}
Keywords

- cognitive impairment

- cognitive rehabilitation

- cognitive retraining

- neuropsychological rehabilitation

- post-concussive symptoms

- traumatic brain injury
\end{abstract}

Objective Traumatic brain injury (TBI) is a leading cause of mortality and chronic disability across the globe. This study aimed to understand the effects of cognitive retraining (CR) intervention on neuropsychological functions, symptom reporting, and quality of life in patients with moderate to severe TBI.

Materials and Methods The present single-group intervention study with a pre-post design included 12 patients diagnosed with moderate to severe TBI within 3-24 months post injury. Outcome measures included National Institute of Mental Health and Neuro Sciences (NIMHANS) Neuropsychology Battery, Perceived Stress Scale, Rivermead Post-Concussion Symptom Questionnaire, World Health Organization Quality of Life Scale-Brief, and Visual Analogue Scale. All patients underwent a total of 20 sessions of hospital-based CR, spanning over a period of 2 months. The CR included tasks targeting to enhance processing speed, attention, executive function, learning, and memory. Outcome assessments were conducted at baseline and immediately at post intervention.

Statistical Analysis Mean, standard deviation, frequency, and percentage were used as measures of descriptive statistics. Pre- and post-intervention scores were compared using Wilcoxon signed-rank test.

Results The results showed that at post intervention, significant improvements were found in processing speed, working memory, planning, visuo-spatial construction, visual memory, and verbal encoding. Subjective symptom reporting, perceived stress, and quality of life in psychological domain also improved.

Conclusion CR can be helpful in improving not only cognition but also symptom reporting and quality of life in moderate to severe TBI.
DOI https://doi.org/ $10.1055 / \mathrm{s}-0041-1722817$ ISSN $0976-3147$.
C 2021. Association for Helping Neurosurgical Sick People.

This is an open access article published by Thieme under the terms of the Creative Commons Attribution-NonDerivative-NonCommercial-License, permitting copying and reproduction so long as the original work is given appropriate credit. Contents may not be used for commercial purposes, or adapted, remixed, transformed or built upon. (https://creativecommons.org/licenses/by-nc-nd/4.0/).

Thieme Medical and Scientific Publishers Pvt. Ltd. A-12, 2nd Floor, Sector 2, Noida-201301 UP, India 


\section{Introduction}

Road traffic injuries are a serious public health challenge worldwide. About 1.35 million deaths result from road traffic injuries each year across the globe and it is the leading cause of deaths among children and young adults. ${ }^{1}$ India has the second highest death rate due to road traffic accidents $(22.6 / 1,00,000)$ in South East Asia. ${ }^{1}$ Road traffic injuries commonly result in traumatic brain injury (TBI) and survivors suffer from chronic disability. Every year, more than one million people are severely disabled due to TBI in India. ${ }^{2}$ Thirty-five percent of patients with TBI continue to experience disability at 1 year. ${ }^{3}$

Posttraumatic chronic disability results from wide range of physical, cognitive, emotional, and behavioral difficulties in survivors. The cognitive sequel of TBI is characterized by impaired attention, memory, processing speed, and executive functions. ${ }^{4}$ There can be emotional and behavioral problems, which include irritability, agitation, aggression, impulsivity, anxiety, stress, and poor social judgment along with psychiatric syndromes such as depression, mania, psychosis, and posttraumatic stress disorder. ${ }^{5}$ These deficits, mainly the cognitive deficits, can disrupt a person's activities of daily routine, occupational functioning, social stability, and their active participation in the society. The health-related quality of life in survivors of TBI has been documented to be adversely affected. People with moderate to severe TBI can have poorer quality of life even 10 years after injury. ${ }^{6}$ Poor quality of life after TBI is commonly associated with chronic stress, somatic symptoms, and posttraumatic psychiatric disorders. ${ }^{7,8}$

The physical, cognitive, emotional, and behavioral disturbances result in impaired activities of daily living, chronic disability, and poor quality of life, and hence neuropsychological rehabilitation plays an important role as it focuses on reducing the disability and improving the quality of life. Cognitive rehabilitation is the major constituent of a neuropsychological rehabilitation program and aims at remediation of posttraumatic cognitive impairments. Various techniques of cognitive rehabilitation such as direct training, strategy training, meta-cognitive training, functional communication skills training, and external memory compensation have been found to be effective and are recommended for use in rehabilitation of patients with TBI. ${ }^{9}$

Cognitive rehabilitation has been documented to be effective in amelioration of cognitive behavioral and emotional disturbances after TBI; however, most of the scientific literature for its effectiveness comes from the western part of the world. There is a lack of cross-cultural research in this area. Neuropsychological interventions have cultural underpinnings. Neuropsychological functions are largely but not exclusively determined by the neural functioning and can be influenced by cultural experiences also. ${ }^{10}$ Scientific literature has shown that post-acute TBI rehabilitation led to poorer outcomes in patients from cultural and linguistic minorities, independent of socioeconomic background, motivation, and participation in rehabilitation. ${ }^{11}$ Therefore, directly importing the well-established methods of neuropsychological rehabilitation from western culture to another may not be justified. A culture-specific evaluation of any neuropsychological intervention is important.

TBI causes significant burden to individual, family, community, and economy in India; however, there is paucity of literature exploring the effectiveness of neuropsychological rehabilitation after TBI. Most of the published work is limited to single-case reports. ${ }^{12-15}$ There is a lack of uniformity in intervention and outcome measures used in these studies. Only limited studies have attempted to explore the effects of neuropsychological rehabilitation in larger sample. Few studies have examined empirically the role of neuropsychological rehabilitation on cognitive and psychosocial outcomes but have taken a mixed sample of TBI, stroke, and hypoxia. ${ }^{16,17}$ Two systematic empirical studies have investigated the use of cognitive symptom management and rehabilitation therapy in patients with TBI, but those were limited to mild TBI only. ${ }^{18,19}$ There is a vast difference between the cognitive outcomes after mild TBI and moderate to severe TBI. ${ }^{20}$ Therefore, evidence from studies on mild TBI cannot be generalized to moderate to severe TBI. A recent study evaluated the effect of home-based computerized neuropsychological rehabilitation for 6 months in patients with moderate to severe TBI. ${ }^{21}$ Results showed significant improvements in cognitive functions and community integration; however, there was no attempt to measure the adherence to treatment, which can have direct impact on outcomes.

There are very few empirical studies in this area. Therefore, the present study aimed at evaluating the effects of structured, hospital-based, cognitive retraining (CR) intervention on neuropsychological functions, symptoms reporting, and quality of life in patients with moderate to severe TBI.

\section{Methods}

\section{Study Design and Sample}

This prospective study with single group pre-post experimental design was conducted in a tertiary care hospital in southern India. Twelve patients aged between 18 and 45 years with a diagnosis of moderate to severe TBI with Glasgow Coma Scale score in the range of 3 to 12 were screened. Right-handed individuals with corrected vision or hearing and postinjury period ranging between 3 and 24 months were included in the study. The patients were excluded if they had mild TBI, or a past history of any psychiatric, neurological, neurosurgical illness, mental retardation, or substance use (except alcohol and nicotine use), or if they had undergone any structured psychological intervention in the past 1 year.

\section{Procedure}

The study was approved by the Institute Ethics Committee and written informed consent was sought from all participants who met inclusion criteria. Sociodemographic and clinical details were obtained. All patients were subjected to baseline assessment, which included a comprehensive 
neuropsychological assessment and assessment of symptoms reporting and quality of life. The assessment of overall functioning of the patient was also taken from the caregivers. All the participants underwent a hospital-based CR intervention for 20 sessions spanning over a period of 2 months. After completion of $\mathrm{CR}$ intervention, outcome assessments were readministered. The outcome measures used in the present study are described below.

\section{Tools}

\section{NIMHANS Neuropsychology Battery ${ }^{22}$}

The cognitive functioning was assessed using NIMHANS Neuropsychology Battery. The Battery consisted of nine tests and covered the cognitive domains of motor speed, processing speed, focused attention, semantic fluency, working memory, planning, visuo-spatial construction, verbal and visual learning, and memory. The Battery has been standardized on Indian population and is widely used for clinical and research purposes. The raw scores were used for the analysis in the present study.

\section{The Rivermead Post-Concussion Symptoms Questionnaire $^{23}$}

This scale is a widely used self-report tool to assess commonly reported post-concussion symptoms. The scale consists of 16 items where each item is rated on a Likert scale ranging from 0 (never experienced at all) to 4 (a severe problem). A total score is obtained by adding up the scores on all items, with higher total score representing more severe post-concussive symptoms.

\section{Perceived Stress Scale ${ }^{24}$}

This is a 10-item self-report measure that assesses the perceived stress. Each item is rated on a Likert scale ranging from 0 (never) to 4 (very often). A total score is obtained by adding up the scores on all items, with higher total score representing higher perceived stress.

\section{World Health Organization Quality of Life Scale-Brief 25} World Health Organization Quality of Life Scale-Brief is a 26-item self-report measure. Each item is rated on a Likert scale ranging from 0 to 5 . The scale assesses the quality of life in four domains, namely physical, psychological, social, and environmental. Total scores are obtained for each domain, with higher scores representing better quality of life.

\section{Visual Analogue Scale}

This was used to assess caregiver's perception of the overall dysfunction of patient. They were asked to rate it on a scale of 0 to 100 where 0 represented adequate functioning and 100 represented complete dysfunction.

\section{Intervention Program: Cognitive Retraining}

The hospital-based CR intervention used in the present study was adapted from the home-based CR developed for patients with head injury by Dr. Shobhini Rao. ${ }^{26}$ The CR intervention was based on the principles of restoration and aimed at enhancement of impaired cognitive functions through intensive and repetitive practice and drills. The CR intervention included five tasks targeting various cognitive domains such as processing speed, attention, response inhibition, working memory, learning, and memory. Each task was designed to have four levels of difficulty arranged in ascending order. Each level was practiced for five sessions before moving onto the next level. The description of the CR intervention is provided in - Table $\mathbf{1}$. Twenty sessions of $\mathrm{CR}$ were performed thrice a week on outpatient basis. Before initiating the CR, each participant along with the caregiver received one session with the therapist focusing on psychoeducation regarding the TBI and its sequel, need for cognitive rehabilitation, and overview of $\mathrm{CR}$ intervention.

\section{Data Analysis}

All the data were entered into the Statistical Package for Social Sciences (IBM-SPSS) software, version 20. Appropriate descriptive statistics such as frequency, percentage, mean, and standard deviation (SD) were used to describe the sociodemographic and clinical data as well as data from the outcome measures. To compare the average scores on various outcome measures between baseline and post intervention, Wilcoxon signed-rank test was used. The level of significance was kept at $5 \%$.

Table 1 A brief description of the cognitive retraining intervention

\begin{tabular}{|l|l|l|l|}
\hline S. no. & Target functions & Task & Description \\
\hline 1 & $\begin{array}{l}\text { Processing speed; } \\
\text { sustained attention }\end{array}$ & Grain sorting & $\begin{array}{l}\text { The patients are given predefined quantity of a mix of two grains and the subject } \\
\text { has to sort the grains separately by sorting one grain at a time in a limited time }\end{array}$ \\
\hline 2 & $\begin{array}{l}\text { Focused attention; } \\
\text { response inhibition }\end{array}$ & Shading & $\begin{array}{l}\text { The patients are given a simple geometrical shape printed on a white plain paper } \\
\text { and the subject evenly shades it without crossing the boundaries in a limited time }\end{array}$ \\
\hline 3 & Working memory & Digit span & $\begin{array}{l}\text { A string of numbers is read to the patient and the patient must repeat it either for- } \\
\text { ward or backward as instructed by the therapist; five items of forward and backward } \\
\text { each were done in every session }\end{array}$ \\
\hline 5 & $\begin{array}{l}\text { Verbal learning and } \\
\text { memory }\end{array}$ & $\begin{array}{l}\text { Temporal } \\
\text { encoding }\end{array}$ & $\begin{array}{l}\text { A list of unrelated words was read out and the subject must repeat the first one-third } \\
\text { of the list; same procedure was done for the last one-third and middle one-third of } \\
\text { the list; free recall learning was done for five trials }\end{array}$ \\
\hline memory & $\begin{array}{l}\text { Three to six small daily use objects such as coin and key, eraser are placed on the } \\
\text { table in predefined spatial arrangement and after an exposure of 10 seconds the } \\
\text { patient must rearrange the jumbled objects in the same spatial arrangement }\end{array}$ \\
\hline
\end{tabular}




\section{Results}

\section{Sociodemographic and Clinical Characteristics of the Sample}

- Table 2 shows the sociodemographic and clinical characteristics of the sample. The age of the patients ranged from 18 to 42 years (mean $\pm S D=32.33 \pm 7.8$ ). The mean age of the sample in the present study is very similar to the findings from another epidemiological study conducted at a Level I trauma center in New Delhi, where the authors reported that mean age of patients with moderate to severe TBI was 32.15 years. ${ }^{27}$ Majority of the participants had studied up till graduation. The years of education ranged from years 6 to 20 (mean $\pm \mathrm{SD}=14.5 \pm 4.12$ ). Most of the participants were male (75\%) and more than half were married (58\%). Most of them were gainfully employed before the injury (83\%) and the rest were students.

All the patients sustained TBI due to road traffic accidents and two-thirds of the patients had sustained a severe TBI (67\%). The duration of illness ranged from 3.1 months to 20.1 months (mean $\pm \mathrm{SD}=11.37 \pm 6.04$ ) at the time of recruitment. Half of the patients presented with complaints of forgetfulness and slowness of thinking whereas other $41 \%$ presented with speech language complaints such as expressive aphasia and anomia.

\section{Effect of CR on Outcome Measures}

The scores obtained on various outcome measures at baseline and at post intervention were compared to assess the immediate post intervention changes in patients.

Table 2 Sociodemographic and clinical characteristics of the sample $(n=12)$

\begin{tabular}{|l|l|}
\hline Variables & $\begin{array}{l}\text { Mean } \pm \text { standard } \\
\text { deviation, or } \boldsymbol{n} \text { (\%) }\end{array}$ \\
\hline Age in years & $32.33 \pm 7.8$ \\
\hline Years of education & $14.5 \pm 4.12$ \\
\hline Gender & $9(75)$ \\
\hline Male & $3(25)$ \\
\hline Female & $7(58)$ \\
\hline Marital status & $4(33)$ \\
\hline Married & $1(9)$ \\
\hline Single & $10(83)$ \\
\hline Separated & $2(17)$ \\
\hline Employment status & $11.37 \pm 6.04$ \\
\hline Employed & $4(33)$ \\
\hline Student & $8(67)$ \\
\hline Duration of illness in months & $6(50)$ \\
\hline Injury severity & $5(41)$ \\
\hline Moderate & $1(9)$ \\
\hline Severe & \\
\hline Presenting complaints & \\
\hline Memory & Language and speech \\
\hline Behavior & \\
\hline
\end{tabular}

\section{Cognitive Functions}

The cognitive functions were assessed using various cognitive tests. - Table 3 shows the comparison of cognitive functions from baseline to post intervention and from post intervention to follow-up. The table shows that patients had significant changes at post intervention in the domains of processing speed (Digit Symbol Substitution Test), working memory (Spatial Span), planning (Tower of London), visuo-spatial construction (Complex Figure Test-Copy), visual memory (Complex Figure Test-Delayed Recall), and verbal memory (Rey's Auditory Verbal Learning Test-Total Learning). It is important to note that on Rey's Auditory Verbal Learning Test (RAVLT), only the scores on Total Learning showed a change whereas there was no significant difference observed on the Delayed Recall scores on this test. Total Learning scores on RAVLT represent the individual's capacity to learn information over trials and is considered as the measures of memory encoding whereas the Recall trials are traditionally considered as the measures of memory retention. Our findings on RAVLT show changes in memory encoding but not in memory retention. The significant difference seen on the test of processing speed (Digit Symbol Substitution Test) in the absence of any significant difference on test of motor speed (Finger Tapping Test) points to the fact that changes in processing speed are independent of any changes in motor speed.

\section{Symptom Reporting, Quality of Life, and Overall Functionality}

- Table 4 shows the comparison of the scores obtained on other outcome measures of the study. It is evident from the table that at post intervention, the patient experienced significantly lower amounts of perceived stress (Perceived Stress Scale), reported lower levels of post-concussive symptoms (Rivermead Post-Concussion Symptoms Scale), and reported higher levels of psychological quality of life. These subjective improvements reported by the patients were also corroborated by the caregivers where they reported significantly lower levels of overall dysfunction in patients (Visual Analogue Scale).

\section{Discussion}

The present study investigated the effects of CR intervention in patients with moderate to severe TBI. The findings of the study reveal significant improvements in the cognitive domains that were trained, that is, processing speed, working memory, visual memory, and verbal memory. These findings are in line with previous researches where participants with acquired brain injury showed significant improvements in the trained cognitive domains, that is, attention, working memory, executive functions, and memory following 8 to 12 weeks of in-hospital computer-based cognitive training. ${ }^{28,29}$ In addition, improvements were seen in the domains of planning and visuo-spatial construction also. Planning and visuo-spatial construction are higher cognitive functions and other basic cognitive functions such as processing speed, attention, working memory, and visualperceptual abilities serve as the building block for these 
Table 3 Comparison of cognitive functions at baseline and at post intervention

\begin{tabular}{|c|c|c|c|c|c|c|c|}
\hline \multirow[t]{2}{*}{ Variables } & \multirow[t]{2}{*}{$n$} & \multicolumn{2}{|c|}{ Baseline } & \multicolumn{2}{|c|}{ Post CR } & \multirow[t]{2}{*}{ z-Value } & \multirow[t]{2}{*}{$p$-Value } \\
\hline & & Mean & SD & Mean & SD & & \\
\hline FTT-Right hand & 10 & 35.98 & 11.96 & 40.9 & 8.954 & -1.201 & 0.240 \\
\hline DSST-Time & 12 & 457.58 & 193.82 & 355.83 & 172.68 & -2.824 & $0.005^{\mathrm{a}}$ \\
\hline Color Trails Test $1-$ Time & 12 & 155.75 & 70.12 & 143.00 & 55.14 & -0.471 & 0.638 \\
\hline Color Trails Test 2-Time & 11 & 309.73 & 204.94 & 270.09 & 140.77 & -1.245 & 0.213 \\
\hline Animals Names test & 11 & 7.55 & 3.05 & 8.27 & 2.45 & -0.9 & 0.368 \\
\hline Verbal 2 Back Hits & 11 & 5.82 & 2.32 & 6.73 & 1.10 & -1.278 & 0.201 \\
\hline Spatial Span & 12 & 14.25 & 2.63 & 15.25 & 2.90 & -1.916 & $0.050^{\mathrm{a}}$ \\
\hline Tower of London & 12 & 7.92 & 1.98 & 10.08 & 1.78 & -2.591 & $0.010^{\mathrm{a}}$ \\
\hline CFT_Copy & 11 & 29.00 & 8.05 & 32.82 & 3.79 & -2.507 & $0.012^{\mathrm{a}}$ \\
\hline CFT_Delayed Recall & 11 & 10.45 & 8.38 & 16.36 & 8.43 & -2.368 & $0.018^{a}$ \\
\hline AVLT_Total Learning & 12 & 31.42 & 13.12 & 37.00 & 12.85 & -2.134 & $0.033^{\mathrm{a}}$ \\
\hline AVLT_Delayed Recall & 12 & 4.83 & 4.04 & 7.00 & 5.10 & -1.838 & 0.066 \\
\hline
\end{tabular}

Abbreviations: RAVLT, Rey's Auditory Verbal Learning Test; CFT, Complex Figure Test; CR, cognitive retraining; DSST, Digit Symbol Substitution Test; FTT, Finger Tapping Test; SD, standard deviation. a $p$-Value significant at 0.05 level.

Table 4 Comparison of symptom reporting, quality of life, and overall functionality at baseline and at post intervention

\begin{tabular}{|c|c|c|c|c|c|c|c|}
\hline \multirow[t]{2}{*}{ Variables } & \multirow[t]{2}{*}{$n$} & \multicolumn{2}{|c|}{ Baseline } & \multicolumn{2}{|c|}{ Post CR } & \multirow[t]{2}{*}{ z-Value } & \multirow[t]{2}{*}{$p$-Value } \\
\hline & & Mean & SD & Mean & SD & & \\
\hline Perceived Stress Scale & 9 & 14.89 & 9.25 & 8.89 & 7.99 & -2.203 & $0.028^{a}$ \\
\hline RPCSQ & 10 & 9.00 & 5.81 & 4.30 & 3.77 & -2.136 & $0.033^{\mathrm{a}}$ \\
\hline WHOQoL-Physical & 9 & 28.89 & 4.48 & 30.22 & 4.49 & -1.130 & 0.258 \\
\hline WHOQoL-Psychological & 9 & 21.44 & 3.88 & 25.22 & 3.19 & -2.120 & $0.034^{\mathrm{a}}$ \\
\hline WHOQoL-Social & 9 & 12.33 & 2.06 & 12.89 & 1.36 & -0.641 & 0.521 \\
\hline WHOQoL-Environmental & 9 & 31.56 & 6.60 & 33.56 & 5.10 & -1.190 & 0.234 \\
\hline Visual Analogue Scale & 12 & 56.25 & 23.85 & 29.58 & 18.52 & -3.103 & $0.002^{\mathrm{a}}$ \\
\hline
\end{tabular}

Abbreviations: CR, cognitive retraining; RPCSQ, Rivermead Post-Concussion Symptom Questionnaire; SD, standard deviation; WHOQoL, World Health Organization Quality of Life Scale-Brief. ${ }^{a} p$-Value significant at 0.05 level.

higher cognitive functions. The improvements observed in planning and visuo-spatial construction in the present study can be attributed to the improvements in basic cognitive functions. Since the focus was on improving the attention, this could have had impact on higher cognitive functions also. Attention training interventions after TBI have been found to be effective for improvement in overall cognitive functions. ${ }^{30}$ Therefore, the improvements in higher cognitive functions indicate toward the generalization or transfer of learning from trained to nontrained domains.

Consistent with the previous literature, the findings further reveal that patients had significant reduction in subjectively reported symptoms, that is, post-concussive symptoms and perceived stress, after $\mathrm{CR}^{29} \mathrm{~A}$ recent randomized controlled trial (RCT) of four CR interventions in military service members with mild TBI reported similar findings where reduced functional cognitive-behavioral difficulties were found in those receiving therapist-administered $C R$ in addition to a combination of psychoeducation and medical management compared with those receiving only the combination of psychoeducation and medical management. ${ }^{31}$ The association between subjectively reported symptoms and cognitive functions in TBI appears bidirectional. The persisting cognitive deficits may be a cause of subjective distress for many individuals with TBI whereas for others, stress and subjective post-concussive symptoms can cause difficulties in cognitive functioning. Somatic symptoms and chronic stress have been found to be significantly associated with subjective memory complaints after mild to moderate TBI. ${ }^{32}$ On the other hand, post-traumatic stress disorder and depression in war veterans with mild TBI have been found to be associated with more enduring cognitive compromise. ${ }^{33}$ The CR intervention used in the present study focused exclusively on cognitive functions. Therefore, improvements in subjective post-concussive symptoms and perceived stress after CR intervention can be understood as the result of cascading effect of improvements in cognitive functions.

Cognitive functions are important for every aspect of human functioning and are the basis of adaptive behavior and holistic functionality in human beings. Cognitive impairments have shown to negatively impact the activities of daily living in brain-injured patients. Present study found 
a significant reduction in overall dysfunction of patients as reported by caregivers after $\mathrm{CR}$ intervention. Cicerone et al found that patients with moderate to severe TBI undergoing intensive cognitive rehabilitation program had better community integration and satisfaction with functioning than those undergoing standard rehabilitation program. ${ }^{34}$ An RCT reported that working memory training in addition to standard neurorehabilitation in patients with brain injury was effective in improving the everyday functioning and reducing the fatigue. ${ }^{35}$ The CR intervention used in the present study has also augmented the recovery of functionality in patients with TBI.

Persons with TBI experience poor health-related quality of life, which is commonly associated with chronic stress, somatic symptoms, and psychiatric disorders with an onset after TBI. ${ }^{7,8}$ In the present study, CR intervention resulted in significant improvement in psychological domain of quality of life whereas other domains such as physical, social, and environmental domains remained unchanged. The improvement seen only in psychological domain of quality of life can be due to the nature of questions in the tools. The questions under psychological domains pertain to intrapersonal factors, for example, mood, concentration, meaning in life, acceptance of self, and self-appearance, whereas the questions in rest of the domains pertain to factors such as physical problems, interpersonal relationships, sex, physical safety, environment, and availability of resources that are beyond one's own direct control. These findings are in line with previous literatures, which also show that cognitive rehabilitation leads to improved quality of life in patients with brain injury. ${ }^{36,37}$

The present study investigated a hospital-based CR intervention whereas literature suggests that home-based cognitive rehabilitation is as good as in-hospital cognitive rehabilitation after moderate to severe TBI. ${ }^{38}$ The home-based cognitive rehabilitation is cost-effective. However, there are few major limitations of home-based cognitive rehabilitation in India. First, in India, with 33\% of population still being illiterate, it is difficult to train the caregivers to function as co-therapist at home for patients and therefore the cognitive rehabilitation plan may not get implemented as systematically as in a hospital-based rehabilitation. Second, measuring compliance to home work in home-based approach is a challenge. Third, it is noteworthy that India has high rates of misconceptions toward the brain damage and brain injury sequel even among the upcoming health-care professionals. ${ }^{39}$ Behavioral and neuropsychiatric disturbances commonly experienced by the survivors of severe TBI may resemble the behavioral disturbances seen in patients with mental illnesses. Abnormal behavior and after-effects of mental illness are major source of stigma in family caregivers of persons with mental illnesses. ${ }^{40}$ Therefore, misconceptions regarding the TBIs and behavioral disturbances after TBI may lead to increased stigma among family caregivers of patients with TBI. This stigma may not let the caregivers carry out the cognitive rehabilitation tasks effectively at home and thereby hampering the outcomes of cognitive rehabilitation. Lastly, hospital-based CR intervention mediated by the therapist may additionally lead to the development of a therapeutic alliance between the patient and therapist, which has been considered a major mechanism of change in most psychological interventions.

To our knowledge, this is the first systematic study to explore the effects of hospital-based cognitive rehabilitation on patients with moderate to severe TBI in India. Structured and intense rehabilitation program and use of combination of objective and subjective outcome measures are the major strengths of the study. The major limitations of the present study include a small sample size, lack of a control group, and short duration of CR intervention. Moreover, the findings from the present study cannot be generalized to the older population with TBI as the sample for the present study consisted of relatively young patients. Further large-scale studies with robust study designs are required to assess the effectiveness of this CR intervention in India. Future studies should also explore the long-term effects of this intervention at follow-up.

\section{Conclusion}

The present preliminary study investigated the effectiveness of a hospital-based, therapist-mediated CR intervention based on the restorative approach of rehabilitation in patients with moderate to severe TBI. The findings from the study are promising as the CR intervention resulted in significant improvements in cognitive functions after 20 sessions of $\mathrm{CR}$ intervention. The $\mathrm{CR}$ intervention also resulted in reduced subjective symptom reporting and enhanced functionality and quality of life. Though the home-based CR interventions are more cost-effective, they may not serve the purpose in patients with severe TBI. The behavioral disturbances and complex disability in survivors of severe TBI may render a home-based CR intervention nonviable and a structured hospital-based CR intervention may be useful.

\section{Ethical Approval}

The present study was approved by the Institute Ethics Committee of National Institute of Mental Health and Neurosciences, Bengaluru.

\section{Funding}

This work was supported by the Cognitive Science Research Initiative (CSRI) of Department of Science and Technology, Government of India (Grant reference: SR/ CSRI/150/2014, dated 03.03.2016).

\section{Conflict of Interest}

None declared.

\section{References}

1 World Health Organization, Global Status Report on Road Safety 2018. Geneva: World Health Organization; 2018

2 Burton A. A key traumatic brain injury initiative in India. Lancet Neurol 2016;15(10):1011-1012 
3 Gururaj G, Kolliri SV, Chandramouli BA, Traumatic Brain Injury. Bengaluru, India: National Institute of Mental Health and Neurosciences Publication; 2005

4 Said PZ, Ghosh A, Pal R. Poli N, Moscote-Salazar LR, Agrawal A. Impact of traumatic brain injury on cognitive functions. Arch Mental Health 2018;19(2):97-101

5 Ahmed S, Venigalla H, Mekala HM, Dar S, Hassan M, Ayub S. Traumatic brain injury and neuropsychiatric complications. Indian J Psychol Med 2017;39(2):114-121

6 Andelic N, Hammergren N, Bautz-Holter E, Sveen U, Brunborg C, Røe C. Functional outcome and health-related quality of life 10 years after moderate-to-severe traumatic brain injury. Acta Neurol Scand 2009;120(1):16-23

7 Bay E, de-Leon MB. Chronic stress and fatigue-related quality of life after mild to moderate traumatic brain injury. J Head Trauma Rehabil 2011;26(5):355-363

8 Diaz AP, Schwarzbold ML, Thais ME, et al. Psychiatric disorders and health-related quality of life after severe traumatic brain injury: a prospective study. J Neurotrauma 2012; 29(6):1029-1037

9 Cicerone KD, Goldin Y, Ganci K, et al. Evidence-based cognitive rehabilitation: systematic review of the literature from 2009 through 2014. Arch Phys Med Rehabil 2019;100(8): 1515-1533

10 Uzzell B, Grasping the cross-cultural reality. In: Uzzell B, Ponton M, Ardilla A, eds. International Handbook of Cross-Cultural Neuropsychology. New Jersey: Lawrence Erlbaum Associates; 2007 1-21

11 Saltapidas H, Ponsford J. The influence of cultural background on motivation for and participation in rehabilitation and outcome following traumatic brain injury. J Head Trauma Rehabil 2007;22(2):132-139

12 Kashyap H, Kumar K. Integrated rehabilitation for a case with traumatic brain injury. Indian J Neurotrauma 2010;7(1):89-91

13 Jamuna N, Pillai S. Home based cognitive retraining in traumatic brain injury. Indian J Neurotrauma 2010;7(1):93-95

14 Mohanty M, Gupta SK. Home based neuropsychological rehabilitation in severe traumatic brain injury: a case report. Ann Neurosci 2013;20(1):31-35

15 Srivastav D, Sharma V, Singh TB. Neuropsychological assessment and cognitive retraining of deficit cognitive functioning in a case of head injury. Indian J Health Wellbeing 2013; 4(6):1357-1359

16 Chopra S, Sinha S, Gupta D, et al. Does functionality cease after acquired brain injury? Vignettes from a neuropsychosocial perspective. Indian J Neurotrauma 2017;14(1):6-14

17 Patil M, Gupta A, Khanna M, et al. Cognitive and functional outcomes following inpatient rehabilitation in patients with acquired brain injury: a prospective follow-up study. J Neurosci Rural Pract 2017;8(3):357-363

18 Kannan S, Chandramohan V, Kannan SR. Efficacy of cognitive intervention using CogSMART in the management of cognitive problems in mild traumatic brain injury. Indian J Health Wellbeing 2017;8(9):1008-1011

19 Kannan S, Kannan R, Chandramohan V. Comprehensive cognitive retraining with CogSMART in mild traumatic brain injury: an interventional study. IP Indian J Neurosci 2019;5:160-166

20 Dikmen SS, Corrigan JD, Levin HS, Machamer J, Stiers W, Weisskopf MG. Cognitive outcome following traumatic brain injury. J Head Trauma Rehabil 2009;24(6):430-438

21 Kanchan A, Singh AR, Khan NA. Jahan M, Raman R, Sathyanarayana Rao TS. Impact of neuropsychological rehabilitation on activities of daily living and community reintegration of patients with traumatic brain injury. Indian J Psychiatry 2018;60(1):38-48

22 Rao SL, Subbakrishna DK, Gopukumar K, NIMHANS Neuropsychology Battery. Bengaluru: National Institute of Mental Health and Neurosciences; 2004
23 King NS, Crawford S, Wenden FJ, Moss NE, Wade DT. The Rivermead Post Concussion Symptoms Questionnaire: a measure of symptoms commonly experienced after head injury and its reliability. J Neurol 1995;242(9):587-592

24 Cohen S, Williamson G, Perceived stress in a probability sample of the United States. In: Spacapan S, Oskamp S, eds. The Social Psychology of Health: Claremont Symposium on Applied Social Psychology. Newbury Park, CA: Sage; 1988 31-67

25 The WHOQOL Group. Development of the World Health Organization WHOQOL-BREF quality of life assessment. Psychol Med 1998;28(3):551-558

26 Rao SL. Home-Based Cognitive Retraining. Paper presented at Second Workshop on Continuing Education in Neuropsychology; 2004; NIMHANS, Bengaluru

27 Kamal VK, Agrawal D, Pandey RM. Epidemiology, clinical characteristics and outcomes of traumatic brain injury: evidences from integrated level 1 trauma center in India. J Neurosci Rural Pract 2016;7(4):515-525

28 Fernández E, Bringas ML, Salazar S, Rodríguez D, García ME, Torres M. Clinical impact of RehaCom software for cognitive rehabilitation of patients with acquired brain injury. MEDICC Rev 2012;14(4):32-35

29 Johansson B, Tornmalm M. Working memory training for patients with acquired brain injury: effects in daily life. Scand J Occup Ther 2012;19(2):176-183

30 Rohling ML, Faust ME, Beverly B, Demakis G. Effectiveness of cognitive rehabilitation following acquired brain injury: a meta-analytic re-examination of Cicerone et al.'s $(2000,2005)$ systematic reviews. Neuropsychology 2009;23(1):20-39

31 Cooper DB, Bowles AO, Kennedy JE, et al. Cognitive rehabilitation for military service members with mild traumatic brain injury: a randomized clinical trial. J Head Trauma Rehabil 2017;32(3):E1-E15

32 Bay E, Kalpakjian C, Giordani B. Determinants of subjective memory complaints in community-dwelling adults with mild-to-moderate traumatic brain injury. Brain Inj 2012;26(7-8):941-949

33 Vasterling JJ, Brailey K, Proctor SP, Kane R, Heeren T, Franz M. Neuropsychological outcomes of mild traumatic brain injury, post-traumatic stress disorder and depression in Iraq-deployed US Army soldiers. Br J Psychiatry 2012;201(3):186-192

34 Cicerone KD, Mott T, Azulay J, Friel JC. Community integration and satisfaction with functioning after intensive cognitive rehabilitation for traumatic brain injury. Arch Phys Med Rehabil 2004;85(6):943-950

35 Björkdahl A, Åkerlund E, Svensson S, Esbjörnsson E. A randomized study of computerized working memory training and effects on functioning in everyday life for patients with brain injury. Brain Inj 2013;27(13-14):1658-1665

36 Cicerone KD, Mott T, Azulay J, et al. A randomized controlled trial of holistic neuropsychologic rehabilitation after traumatic brain injury. Arch Phys Med Rehabil 2008;89(12):2239-2249

37 Svendsen HA, Teasdale TW. The influence of neuropsychological rehabilitation on symptomatology and quality of life following brain injury: a controlled long-term follow-up. Brain Inj 2006;20(12):1295-1306

38 Salazar AM, Warden DL, Schwab K, et al; Defense and Veterans Head Injury Program (DVHIP) Study Group. Cognitive rehabilitation for traumatic brain injury: a randomized trial. JAMA 2000;283(23):3075-3081

39 Gurusamy J, Gandhi S, Amudhan S, et al. Misconceptions about traumatic brain injury among nursing students in India: implications for nursing care and curriculum. BMC Nurs 2019; 18(1):64-73

40 Mehrotra K, Nautiyal S, Raguram A. Mental health literacy in family caregivers: a comparative analysis. Asian J Psychiatr 2018;31:58-62 